\title{
Executive function in body dysmorphic disorder
}

\author{
J. Dunai ${ }^{1}$, I. Labuschagne ${ }^{2}$, D. J. Castle ${ }^{1,3,4}$, M. Kyrios ${ }^{5}$ and S. L. Rossell ${ }^{1,2,4 *}$ \\ ${ }^{1}$ School of Behavioural Science, Department of Psychology, University of Melbourne, Melbourne, Australia \\ ${ }^{2}$ MAP-RC, School of Psychology, Psychiatry and Psychological Medicine, Faculty of Medicine, Nursing and Health Sciences, Monash University, \\ Melbourne, Australia \\ ${ }^{3}$ Department of Psychiatry, St Vincent's Hospital, Melbourne, Australia \\ ${ }^{4}$ Mental Health Research Institute of Victoria, Melbourne, Australia \\ ${ }^{5}$ Department of Psychology, Swinburne University, Melbourne, Australia
}

Background. Body dysmorphic disorder (BDD) is a poorly understood disorder that involves a preoccupation with imagined or minor bodily defects. Only a few studies of neuropsychological function have been conducted. Two previous studies have indicated executive dysfunction in BDD. The current study sought to further define these executive deficits.

Method. Fourteen DSM-IV BDD patients and 14 age- and sex-matched control participants took part. Because of the high incidence of co-morbidity in BDD, patients with co-morbid Axis I disorders were not excluded. Control participants had no history of psychiatric illness. All participants completed the following executive function (EF) tests: Spatial Span (SS), Spatial Working Memory (SWM) and the Stockings of Cambridge (SOC) task. They also completed the Pattern Recognition (PR) test, a test of visual memory (VM).

Results. BDD participants made significantly more between-search errors on the SWM task, an effect that increased with task difficulty. Between-search errors are an example of poor maintenance and manipulation of information. SOC results indicated slower subsequent thinking times (i.e. the time taken to plan) in BDD participants. There were no group differences in SS or PR scores. The severity of BDD, depressive or anxiety symptoms was not correlated with performance on any of the cognitive tasks.

Conclusions. The results of this study indicate that BDD patients have EF deficits in on-line manipulation, planning and organization of information. By contrast, spatial memory capacity, motor speed and visual memory were intact. Considered with evidence from lesion and neuroimaging studies, these results suggest frontal lobe dysfunction in BDD.

Received 2 July 2008; Revised 23 October 2009; Accepted 23 October 2009; First published online 2 December 2009

Key words: Body dysmorphic disorder, executive function.

\section{Introduction}

Body dysmorphic disorder (BDD) is characterized by a preoccupation with imagined or minor defects in appearance leading to significant distress and impairment in social and/or occupational functioning. Preoccupations commonly concern face, skin and hair (e.g. Phillips, 2000). BDD is frequently co-morbid with obsessive-compulsive disorder (OCD) and the two disorders share many features. Individuals with BDD experience recurrent, persistent and intrusive preoccupations about their perceived physical defects that are similar to the obsessions seen in OCD. Ritualistic BDD behaviours such as mirror checking

* Address for correspondence: A/Professor S. L. Rossell, Cognitive Neuropsychiatry Laboratory, MAP-RC, School of Psychology, Psychiatry and Psychological Medicine, 1st Floor, Old Baker Building, The Alfred, Commercial Road, Melbourne, VIC 3004, Australia. (Email : SRossell@SRossell.com) and hair grooming are similar to the compulsions of OCD. For these reasons it has been speculated that BDD may be an obsessive-compulsive spectrum disorder (OCSD; see review by Castle et al. 2006).

Little is known about cognitive impairments associated with BDD. To date, there have been two published neuropsychological studies of BDD. Hanes (1998) compared 14 BDD, 10 OCD and 24 control participants and observed impaired performance on the New Tower of London (NTOL) and the Stroop test in both BDD and OCD patients. Deckersbach et al. (2000b) compared 17 BDD participants with 17 control participants on the Rey Complex Figure Test (RCFT) and the California Verbal Learning Test (CVLT). BDD participants showed deficits in verbal (CVLT) and visual (RCFT) memory indices that were mediated by deficits in the use of organizational strategies, suggesting impaired executive function (EF). A previous study observed similar results in 17 OCD participants 
(Deckersbach et al. 2000a). Both these studies suggest that BDD involves executive and frontal system dysfunction, and support a link between BDD and OCD. However, their results are difficult to compare as they used different test batteries and obtained conflicting results where similar tests were used. For example, Hanes (1998) found no deficits in BDD on the RCFT and RALVT whereas Deckersbach et al. (2000b) observed deficits in immediate recall for the RCFT and CVLT. Thus, although it seems that BDD probably involves EF deficits, further research is needed to elucidate the precise pattern of cognitive deficits that characterize the disorder. Such research is important because EF deficits may explain why BDD patients tend to focus more on specific details as opposed to scanning, for example, the whole face.

The aim of the current project was to further explore EF deficits in BDD. In addition, a test of visual memory (VM) not mediated by organizational strategies was included in the study to assist in the identification of 'pure' VM deficits associated with BDD. It was predicted that BDD patients would not show pure VM deficits on this task, suggesting that Deckersbach's VM findings were due to EF problems. The relationship between symptom severity and EF deficits was also explored.

\section{Method}

\section{Participants}

Fourteen participants (nine females, five males) who met DSM-IV criteria for BDD (APA, 2000) and 14 ageand sex-matched control participants took part in the study. BDD participants were recruited from a specialist BDD out-patient clinic (author D.J.C.). For all patients, BDD was the primary problem as determined by their treating psychiatrist and chart review. DSM-IV diagnosis was made using the Body Dysmorphic Disorder Module (Phillips et al. 1995), a reliable diagnostic module modelled after the SCID. Control participants were recruited from a Control Participants Register. Exclusion criteria for all participants included major medical or neurological illness and head injury. The Anxiety Disorders Interview Schedule for DSM-IV (ADIS-IV; Brown et al. 1994) was administered to all participants to identify current Axis I diagnoses. Controls with a history of psychiatric illness and/or alcohol or substance abuse (determined from the ADIS-IV and the control screen from the SCID; First et al. 1996) were excluded from the study. Because of the high incidence of co-morbidity in BDD, patients with co-morbid Axis I disorders (determined by the ADIS and chart review) were not excluded from the study as this would not have produced a representative sample. Patients experienced an average of $1.9 \pm 1.6$ co-morbid disorders [major depressive disorder $(n=7)$, social phobia $(n=5)$, generalized anxiety disorder $(n=4)$, panic disorder $(n=3)$ and OCD $(n=3)]$. Two patients had not been prescribed psychotropic medication, three were taking antidepressants, one was taking an antipsychotic, six were taking antidepressants and antipsychotics, one was taking antidepressant, antipsychotic and anti-addictive medication and one was taking antidepressant and anxiolytic medication. Axis II disorders were not assessed. Clinical assessments were not videotaped but were discussed informally with other members of the research team. The University of Melbourne Human Research Ethics Committee approved the study. All participants provided written informed consent.

\section{Screening instruments}

Participants were assessed for the presence and severity of OCD and BDD symptoms using the Yale-Brown Obsessive Compulsive Scale (YBOCS; Goodman et al. 1989) and the YBOCS modified for BDD (BDD-YBOCS; Phillips et al. 1997) respectively. The Beck Depression Inventory, version 1 (BDI-1; Beck et al. 1961) and the Beck Anxiety Inventory (BAI; Beck et al. 1988) were administered to both BDD and control participants to assess the level of depressive and anxiety symptomatology. The National Adult Reading Test (NART; Nelson, 1982) was used to assess IQ.

\section{Cognitive task battery}

Participants also completed three EF tests and one VM test, selected from the Cambridge Neuropsychological Test Automated Battery (CANTAB). These tests were chosen because they have been used extensively in patients with OCD and known or suspected EF deficits (Owen et al. 1990; Fray et al. 1996). These are described briefly below.

Spatial Span (SS). This test measures spatial short-term memory span. For each trial participants observe a sequence of white squares, some or all of which change colour one by one. Participants are required to remember the location and sequential order of squares that change colour. Spatial span is defined as the highest number of squares for which at least one sequence is recalled correctly. Possible scores range from 0 to 9 .

Spatial Working Memory (SWM). In the SWM test participants are required to search the screen for tokens hidden inside boxes. There are four levels of 
difficulty: three, four, six or eight box searches. Three scores are obtained. A between-search error involves returning to search a box in which a token has already been found. A within-search error involves searching any box more than once during a sequence. Strategy scores reflect how often search sequences are initiated from the same box within a trial, with higher scores indicating many searches starting from different boxes and poor strategy use.

Stockings of Cambridge (SOC). This test assesses spatial planning ability. Participants are required to rearrange a set of balls in the bottom half of the screen to match the positions of balls presented in the top half of the screen. The minimum number of moves to solution is two, three, four or five moves. Accuracy measures during the copying trials consist of the total number of trials completed, the number of trials completed in the minimum number of moves, and the total number of moves in excess of the minimum.

For each copying trial, a control 'following' condition is used to provide a measure of motor initiation and subsequent execution times that are independent of thinking times. Following trials are exact replications of participants' earlier copying moves. The measurement of initiation and subsequent execution latencies in the following condition provides estimates of motor speed. Estimates of cognitive speed are obtained by subtracting following times from total copying times. Thus, the programme provides initial and subsequent movement (following) and thinking times, in addition to the accuracy measures listed above.

Pattern Recognition (PR). This test assesses visual pattern recognition memory. Participants view two target sets of 12 abstract patterns. Each set is followed by 12 pairs of patterns, a target and a distracter. Participants are required to point to the target. Possible scores range from $0 \%$ to $100 \%$.

\section{Statistical methods}

The statistical analyses for each of the four tasks were as follows:

(1) SS and PR performance was compared between groups using a one-way ANOVA.

(2) Performance on the SWM task (number of between-search and within-search errors) was compared between groups using two group by task difficulty repeated-measures ANOVAs. SWM strategy scores and total numbers of between- and within-search errors were compared using oneway ANOVAs.

(3) Latencies for the SOC task were recorded in milliseconds and transformed into fifth roots to reduce skewness in the distribution. Following (movement) and thinking latencies were compared between groups using a group by component (following versus thinking) by timing (initiation versus subsequent) by task difficulty repeated-measures ANOVA. SOC accuracy measures were compared between groups using oneway ANOVAs.

For (1) and (2) significant interaction effects were investigated using Newman-Keuls post-hoc tests. Several of the tasks yielded multiple measures (which are likely to be correlated), thus Bonferroni correction (which assumes that comparisons are independent) was considered too conservative. The error rate required to demonstrate significance was therefore set at 0.01 for comparisons of group differences.

Pearson's product-moment correlation coefficients were calculated to examine the relationship between demographic and screening measures (age, years of education, BDI-1, BAI, BDD-YBOCS, YBOCS and NARTIQ) and performance on the cognitive task battery. Correlational analyses were also used to examine inter-relationships between cognitive task battery tests. The error rate required to demonstrate significance for correlational analyses was set at 0.01 .

\section{Results}

\section{Screening instruments}

Screening data for BDD and control participants are shown in Table 1. There were no significant differences in age and years of education. BDD participants had significantly higher BDI-1, BAI and NARTIQ scores. There were no significant correlations between IQ, depression or anxiety and any of the cognitive task measures, nor did the direction or significance of the ANOVA results change if these variables were included in the model. Based on the BDD-YBOCS, nine out of 14 patients had scores $>20$, indicating current clinically significant BDD. One patient had a score of 19 , four had scores $>15$.

\section{Cognitive task battery}

SS. There was a non-significant trend for BDD patients to have lower SS scores than control participants $[F(1,25)=5.1, \quad p=0.03$, effect size $(E S)=0.17] \quad$ (see Table 2).

SWM. There were significant main effects of group $[F(1,26)=10.6, p=0.003, \mathrm{ES}=0.29] \quad(\mathrm{BDD}=7.8$ and controls $=3.1)$ and task difficulty $[F(1,26)=49.6$, $p<0.0005, \mathrm{ES}=0.66$ ] (Level: three $=0.04$, four $=0.75$, 
Table 1. Screening data for BDD and control participants

\begin{tabular}{lcccl}
\hline & BDD & \multicolumn{2}{c}{ Group comparisons } \\
\cline { 4 - 5 } & Mean (s.D.) & $\begin{array}{l}\text { Control } \\
\text { Mean (s.D.) }\end{array}$ & F value & $p$ \\
\hline Age (years) & $32.9(13.5)$ & $32.8(13.6)$ & 0.001 & 0.9 \\
Years of education & $14.7(3.8)$ & $16.2(3.8)$ & 1.0 & 0.3 \\
BDI-1 & $17.6(11.0)$ & $2.1(2.2)$ & 27.0 & 0.0005 \\
BAI & $19.6(8.7)$ & $5.4(5.5)$ & 27.1 & 0.0005 \\
BDD-YBOCS & $23.8(10.0)$ & $0(0)$ & 79.8 & 0.0005 \\
YBOCS & $5.4(9.2)$ & $0(0)$ & 4.9 & 0.03 \\
NARTIQ & $112.6(7.6)$ & $119.0(4.8)$ & 7.0 & 0.01 \\
\hline
\end{tabular}

BDD, Body dysmorphic disorder; BDI-1, Beck Depression Inventory, version 1; BAI, Beck Anxiety Inventory; BDD-YBOCS, Yale-Brown Obsessive Compulsive Scale modified for BDD; YBOCS, Yale-Brown Obsessive Compulsive Scale; NARTIQ, National Adult Reading Test-estimated verbal IQ; S.D., standard deviation.

Table 2. Performance on the task battery in BDD and control participants

\begin{tabular}{lcccc}
\hline & & & \multicolumn{2}{c}{ Group comparisons } \\
\cline { 3 - 5 } Measure & BDD & Control \\
Mean (s.D.) & Mean (s.D.) & F value & $p$ \\
\hline Spatial Span (SS) & $6.1(1.2)$ & $7.1(1.3)$ & 5.1 & 0.03 \\
Spatial Working Memory (SWM) & & & \\
$\quad$ Total between-search errors & $31.4(19.3)$ & $12.2(10.7)$ & 10.6 & 0.003 \\
$\quad$ Total within-search errors & $2.6(4.2)$ & $1.5(2.3)$ & 0.7 & 0.4 \\
$\quad$ Strategy score & $33.6(6.5)$ & $30.1(6.2)$ & 2.0 & 0.2 \\
Stockings of Cambridge (SOC) & & & & 0.005 \\
$\quad$ Number of problems solved & $10.1(1.3)$ & $11.3(0.7)$ & 9.7 & 0.003 \\
$\quad$ Number of perfect solutions & $6.5(1.1)$ & $8.4(1.8)$ & 10.7 & 0.0005 \\
$\quad$ Total moves in excess of minimum & $20.6(5.4)$ & $11.6(6.4)$ & 16.3 & 0.5 \\
Pattern Recognition (PR) & $87.5(10.7)$ & $87.2(12.1)$ & 0.4 & 0.5 \\
\hline
\end{tabular}

BDD, Body dysmorphic disorder; s.D., standard deviation.

six $=5.21$ and eight $=15.79)$, and a significant group by task difficulty interaction $[F(1,26)=8.3, p=0.008, \mathrm{ES}=$ $0.24]$. The BDD participants made significantly more between-search errors than controls as shown in Fig. 1a. Post-hoc tests revealed that the effect of task difficulty was greater in BDD patients, who made significantly more errors at difficulty level eight (but no other level) compared to controls $(p<0.05)$. The within-search errors ANOVA did not show a difference between groups $[F(1,26)=0.7, p=0.4$, $\mathrm{ES}=0.03]$ (see Table 2, Fig. 1b). There was a trend towards increasing numbers of within-search errors as task difficulty increased $[F(1,26)=7.0, p=0.01, \quad E S=0.21]$ (Level: three $=0.04$, four $=0.04$, six $=0.18$ and eight $=$ 1.79), but no significant group by task difficulty interaction $[F(1,26)=1.5, p=0.2, \mathrm{ES}=0.05]$ (see Fig. 1b). There was no significant group difference in SWM strategy scores $[F(1,26)=2.0, p=0.2, \mathrm{ES}=0.07]$ (see Table 2).

Using Pearson's $r$ there was a significant relationship between the number of between-search errors and the extent to which a systematic strategy was used in $\operatorname{BDD}(r=0.74, p=0.003)$ but not in controls $(r=0.6$, $p=0.02)$. To examine the effect of short-term memory capacity on working memory performance, correlations between SS and SWM measures were performed. For controls there was a significant correlation between SS and strategy scores $(r=-0.75, p=0.002)$, indicating that high SS scores were associated with better strategy use, but no significant relationship between SS and number of between-search errors. For BDD patients there were non-significant trends for higher SS scores to be associated with lower (better) strategy scores $(r=-0.64, p=0.01)$ and 
(a)

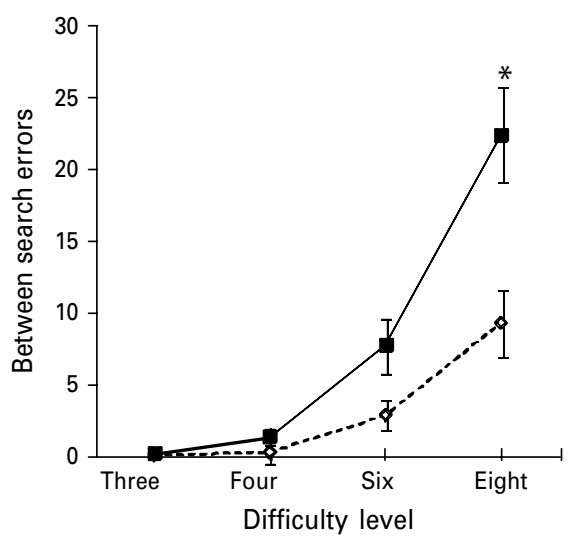

(b)

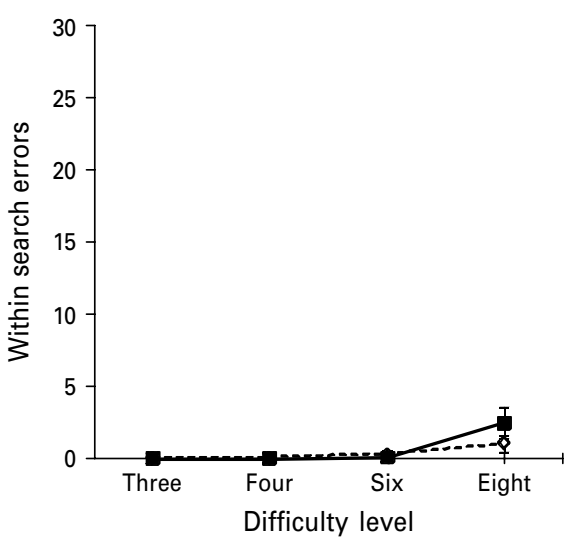

Fig. 1. Data from the spatial working memory task. (a) Number of between-search errors and $(b)$ number of within-search errors. Difficulty level refers to the number of boxes to be searched for tokens. Bars indicate standard errors $\left({ }^{*} p<0.05\right)$. - - - - , Control ; - $\mathbf{-}-$, body dysmorphic disorder.

(a)
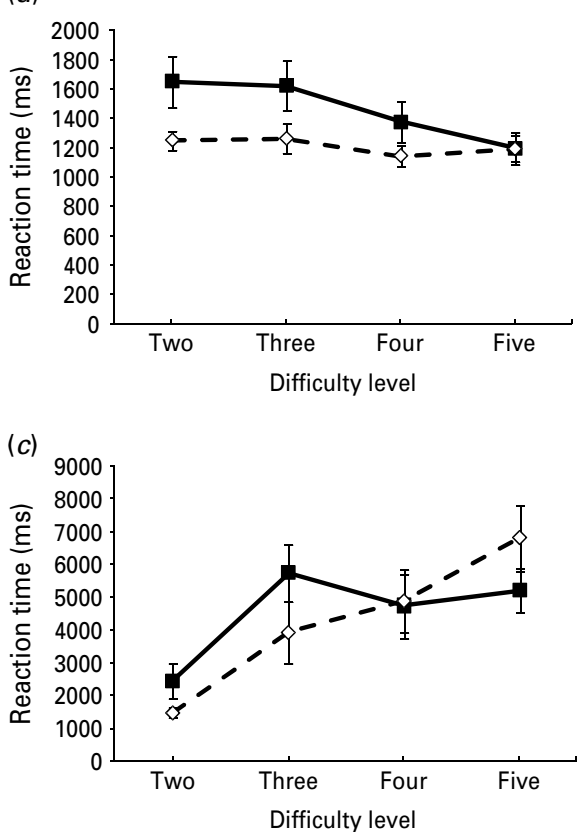

(b)
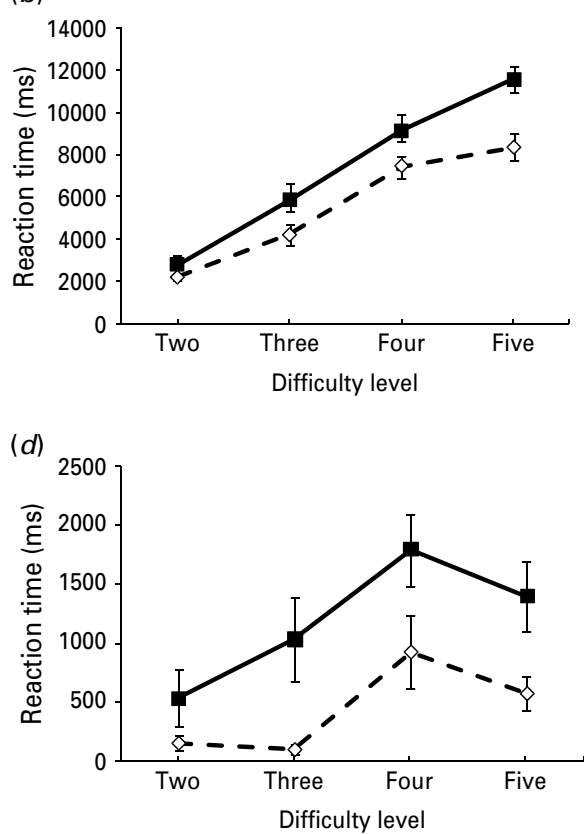

Fig. 2. Data from the Stockings of Cambridge task. (a) Following initiation time; (b) following subsequent time; (c) thinking initiation time; $(d)$ thinking subsequent time. Difficulty level refers to the minimum number of moves required to solve a problem. Raw data are presented in this figure for ease of interpretation. Bars indicate standard errors. - - $\bigcirc-$ - , Control;

- - , body dysmorphic disorder.

decreasing numbers of between-search errors $(r=$ $-0.61, p=0.02)$.

SOC. BDD participants solved significantly fewer problems overall, solved significantly fewer problems in the minimum number of moves, and made significantly more moves in excess of the minimum (see Table 2). In addition, BDD patients demonstrated significant deficits in thinking speed. This is evident from Fig. 2, which shows following (movement) and thinking latencies as a function of timing (initiation or subsequent time) and difficulty level (two-move, three-move, four-move or five-move solutions). The results of a group by component (following or thinking) by timing (initiation or subsequent) by difficulty level ANOVA revealed that there was a significant main effect of group, indicating that, overall, BDD patients were slower than controls $[F(1,26)=$ $10.5, p=0.003, \mathrm{ES}=0.29](\mathrm{BDD}=4.6$ and $\mathrm{Control}=4.2)$. There were also main effects of component, timing and 
difficulty level. For all participants following movement latencies were slower than thinking latencies $[F(1,26)=76.0, p<0.0005, \mathrm{ES}=0.75] \quad$ (following $=4.9$ and thinking $=3.9)$; initiation latencies were slower than subsequent latencies $[F(1,26)=25.9, p<0.0005$, $\mathrm{ES}=0.5$ ] (initiation $=4.6$ and subsequent $=4.2$ ); and latencies increased with increasing level of task difficulty $[F(1,26)=45.5, p<0.0005, E S=0.64]$ (Moves: two $=3.8$, three $=4.2$, four $=4.8$ and $5=4.8$ ). There were also several significant interactions. There was a significant group by timing interaction $[F(1,26)=12.6$, $p=0.001, \mathrm{ES}=0.33$ ]. Post-hoc tests $(p<0.05)$ revealed that initiation latencies were similar between the two groups $(\mathrm{BDD}=4.7$ and Control $=4.6)$, but controls had significantly faster subsequent latencies than BDD participants $(\mathrm{BDD}=4.6$ and control $=3.8)$. There was also a component by timing by group interaction $[F(1,26)=8.4, p=0.008, \mathrm{ES}=0.24]$, indicating that the group by timing interaction was the result of faster latencies in control participants for subsequent thinking times. Post-hoc tests (Newman-Keuls, $p<0.05$ ) confirmed that latencies for all component by timing combinations were similar in both groups except for subsequent thinking times, when controls were faster than BDD participants (subsequent thinking: BDD = 3.4 and Controls $=2.0$ ). This is evident from Fig. $2 d$. In summary, the major difference between the groups was slower subsequent thinking times in BDD participants compared to controls.

$P R$. There were no significant group differences in PR scores $[F(1,24)=0.4, p=0.5]$.

\section{Correlations between demographic/screening measures and performance on cognitive tests}

Years of education, BDI-1, BAI, BDD-YBOCS and NARTIQ were not significantly correlated with any CANTAB measures in either BDD or control participants. Age was significantly correlated with SOC average initiation following (movement) times in BDD and control participants $(r=0.71, p=0.005$ and $r=0.7$, $p=0.007$ respectively), and with SOC average subsequent following times in BDD, but not controls ( $r=0.7, p=0.009$ and $r=0.6, p=0.04$ respectively). This suggests slower movement times with increasing age, particularly in BDD patients.

\section{Discussion}

The results demonstrate that in comparison to ageand sex-matched controls, patients with BDD exhibit deficits in EF related to spatial working memory and subsequent thinking speed. By contrast, on tasks assessing short-term memory capacity, motor speed and visual memory, patients' performance was similar to controls. There was no relationship between symptom severity and performance on any of the cognitive tasks. Similarly, although BDD patients experienced significantly more depressive and anxiety symptoms, and tended to have lower NARTIQ scores than controls, there was no relationship between these measures and performance on cognitive tasks. Although the correlational nature of these later findings is acknowledged, they suggest the absence of significant influences of depression, anxiety or IQ on group differences in cognitive performance, thus indicating that the EF deficits observed in the present study are related to the clinical features of this sample of BDD patients. These findings are broadly consistent with those of Hanes (1998) and Deckersbach et al. (2000b), who both reported EF deficits in BDD that were similar to those observed in OCD. However, direct comparison with these two studies is difficult because different tests of EF were used.

BDD patients' spatial short-term memory capacity (indicated by SS scores) was not significantly compromised, suggesting that the ability to hold spatial information 'on-line' is not reduced in BDD, although further research is required given the trend for lower SS scores in BDD. To date, no other neuropsychological study of BDD has assessed SS. However, Owen et al. (1990) also observed normal SS in patients with unilateral or bilateral frontal lobe excisions, suggesting that short-term memory deficits are not mediated by frontal regions. Despite having (near) normal SS, when required to manipulate increasing amounts of spatial information in the SWM task, BDD participants made significantly more between-search errors than controls. Between-search errors indicate difficulty manipulating on-line information. Group differences increased as task difficulty increased; however, this did not seem to be related to poor strategy use as there were no significant differences between BDD and control strategy scores. The two groups also seemed to rely to a similar extent on short-term memory capacity during performance of the SWM task. This is suggested by significant or near-significant correlations between SS and SWM strategy scores in both groups. Finally, BDD patients' preserved ability to avoid within-search errors indicates some ability to use online processing to manipulate spatial information. As with SS, this is the first study to have examined spatial working memory in BDD patients.

The results of the SOC task indicated that higher level planning ability was impaired in patients with BDD. Compared to controls, patients solved significantly fewer problems overall, solved significantly fewer problems in the minimum number of moves, and made significantly more moves in excess of the 
minimum required to solve a problem. In addition, BDD patients had significantly slower subsequent thinking times compared to controls. This pattern has been observed in schizophrenia (Pantelis et al. 1997) and OCD (Veale et al. 1996), and is thought to be a consequence of poor initial planning leading to mistakes and pauses for further planning. As noted above, impaired performance on the NTOL (the test from which the SOC was developed) has also been observed in both BDD and OCD patients, whose performance did not differ (Hanes, 1998). Poor planning and mistakes on the SOC task could be due to BDD patients' decreased ability to use on-line processing to manipulate spatial information (indicated by spatial working memory deficits); however, there was no correlation between any spatial working memory index and measures of SOC accuracy, movement or thinking times.

From a neurocognitive perspective, the EF deficits observed in the present study suggest frontal involvement in BDD, which is consistent with the idea of BDD as an OCSD involving frontal-striatal dysfunction (Maruff et al. 2002), and with the neurobiological model of BDD described by Saxena \& Feusner (2006). As indicated above, a similar pattern of deficits has been observed in other disorders with presumed frontal dysfunction. However, as suggested by Purcell et al. (1998), these deficits could also be due to cognitive processes (e.g. suppression of unwanted thoughts, uncertainty related to internal representations) that are not necessarily neurocognitive in nature but that nevertheless affect neuropsychological performance.

\section{Clinical implications}

To date, both pharmacological (e.g. selective serotonin reuptake inhibitors, SSRIs) and psychological (e.g. exposure and response prevention, cognitive therapy or cognitive behavioural therapy) treatments for OCD have proved effective in BDD (see, for example, review by Castle \& Rossell, 2006). Additional treatment implications are related to the neuropsychological findings of this study. First, the identification of neurocognitive deficits characteristic of BDD may contribute to localization of specific underlying brain regions, which in turn may lead to the development of more targeted treatments (i.e. brain stimulation). Second, identification of neurocognitive deficits may lead to the development of more effective cognitive and/or behavioural treatments designed to improve, or compensate for, these deficits.

\section{Methodological limitations}

A major limitation was the small sample size. In particular, there were some non-significant trends that may have achieved statistical significance with a larger sample size (as indicated by large ES). These included SS, SWM strategy scores and SOC initial and subsequent following (movement) times. Importantly, the EF deficits were not related to BDD symptoms as obtained from the BDD-YBOCS scores, suggesting that the current data are the result of trait differences in the BDD cohort. However, the current BDD group had low BDD-YBOCS scores, again indicating the importance of examining a larger group with a greater range of BDD-YBOCS scores. A final limitation is the inclusion of patients with co-morbid OCD, which could explain the observed similarity between BDD and OCD. However, given that only three of the 14 BDD patients had co-morbid OCD, this interpretation is unlikely.

\section{Conclusions}

In summary, BDD patients were found to have normal short-term memory capacity and visual memory. By contrast, significant deficits in spatial working memory and cognitive speed during planning tasks were observed. This pattern of findings is consistent with the conceptualization of BDD as an OCSD.

\section{Acknowledgements}

This paper was undertaken as part of an unfunded project at the University of Melbourne.

\section{Declaration of Interest}

None.

\section{References}

APA (2000). Diagnostic and Statistical Manual of Mental Disorders, 4th edn, text revision (DSM-IV-TR). American Psychiatric Association: Washington, DC.

Beck AT, Epstein N, Brown G, Steer RA (1988). An inventory for measuring clinical anxiety: psychometric properties. Journal of Consulting and Clinical Psychology 56, 893-897.

Beck AT, Ward C, Mendelson M (1961). Beck Depression Inventory (BDI). Archives of General Psychiatry 4, 561-571.

Brown TA, DiNardo PA, Barlow DH (1994). The Anxiety Disorders Interview Schedule for DSM-IV. Greywind Publications Inc: New York.

Castle DJ, Rossell S (2006). An update on body dysmorphic disorder. Current Opinion in Psychiatry 19, 74-78.

Castle DJ, Rossell S, Kyrios M (2006). Body dysmorphic disorder. Psychiatric Clinics of North America 29, 521-538.

Deckersbach T, Otto MW, Savage CR, Baer L, Jenike MA $(2000 a)$. The relationship between semantic organization 
and memory in obsessive-compulsive disorder.

Psychotherapy and Psychosomatics 69, 101-107.

Deckersbach T, Savage CR, Phillips KA, Wilhelm S,

Buhlmann U, Rauch SL, Baer L, Jenike MA (2000b).

Characteristics of memory dysfunction in body dysmorphic disorder. Journal of the International Neuropsychological Society 6, 673-681.

First MB, Spitzer RL, Gibbon M, Williams JB (1996). Structured Clinical Interview for DSM-IV Axis I Disorders, Clinician Version (SCID-CV). American Psychiatric Press: Washington, DC.

Fray P, Robbins T, Sahakian B (1996). Neuropsychiatric applications of CANTAB. International Journal of Geriatric Psychiatry 11, 329-336.

Goodman WK, Price LH, Rasmussen SA, Mazure C, Fleischmann RL, Hill CL, Heninger GR, Charney DS (1989). The Yale-Brown Obsessive Compulsive Scale. I. Development, use, and reliability. Archives of General Psychiatry 46, 1006-1011.

Hanes KR (1998). Neuropsychological performance in body dysmorphic disorder. Journal of the International Neuropsychological Society 4, 167-171.

Maruff P, Purcell R, Pantelis C (2002). Obsessive-compulsive disorder. In Cognitive Deficits in Brain Disorders (ed. J. E. Harrison and A. M. Owen). Martin Dunitz: London.

Nelson HE (1982). National Adult Reading Test (NART): Test Manual. NFER-Nelson: Windsor, UK.

Owen A, Downes J, Sahakian B, Polkey C, Robbins T (1990). Planning and spatial working memory following frontal lobe lesions in man. Neuropsychologia 28, 1021-1034.

Pantelis C, Barnes T, Nelson H, Tanner S, Weatherley L, Owen A, Robbins T (1997). Frontal-striatal cognitive deficits in patients with chronic schizophrenia. Brain 120, 1823-1843.

Phillips KA (2000). Quality of life for patients with body dysmorphic disorder. Journal of Nervous and Mental Disease 188, 170-175.

Phillips KA, Atala K, Pope H (1995). Diagnostic instruments for body dysmorphic disorder. In New Research Program and Abstracts. American Psychiatric Association 148th Annual Meeting, Miami, FL.

Phillips KA, Hollander E, Rasmussen SA, Aronowitz BR, DeCaria C, Goodman WK (1997). A severity rating scale for body dysmorphic disorder: development, reliability and validity of a modified version of the Yale-Brown Obsessive Compulsive Scale. Psychopharmacology Bulletin 33, 17-22.

Purcell R, Maruff P, Kyrios M, Pantelis C (1998). Cognitive deficits in obsessive-compulsive disorder on tests of frontal-striatal function. Biological Psychiatry 43, 348-357.

Saxena S, Feusner J (2006). Toward a neurobiology of body dysmorphic disorder. Primary Psychiatry 13, 41-48.

Veale D, Sahakian B, Owen A, Marks I (1996). Specific cognitive deficits in tests sensitive to frontal lobe dysfunction in obsessive-compulsive disorder. Psychological Medicine 26, 1261-1269. 


\section{University Library}

\section{- M M I N E R VA A gateway to Melbourne's research publications}

Minerva Access is the Institutional Repository of The University of Melbourne

Author/s:

Dunai, J;Labuschagne, I;Castle, DJ;Kyrios, M;Rossell, SL

Title:

Executive function in body dysmorphic disorder

Date:

2010-09-01

Citation:

Dunai, J., Labuschagne, I., Castle, D. J., Kyrios, M. \& Rossell, S. L. (2010). Executive function in body dysmorphic disorder. PSYCHOLOGICAL MEDICINE, 40 (9), pp.1541-1548. https://doi.org/10.1017/S003329170999198X.

Publication Status:

Published

Persistent Link:

http://hdl.handle.net/11343/33026 